\title{
Selective Linear or Quadratic Optomechanical Coupling via Measurement
}

\author{
Michael R. Vanner \\ Vienna Center for Quantum Science and Technology (VCQ), Faculty of Physics, University of Vienna, \\ Boltzmanngasse 5, Vienna A-1090, Austria
}

(Received 3 June 2011; published 28 November 2011)

\begin{abstract}
The ability to engineer both linear and nonlinear coupling with a mechanical resonator is an important goal for the preparation and investigation of macroscopic mechanical quantum behavior. In this work, a measurement based scheme is presented where linear or square mechanical-displacement coupling can be achieved using the optomechanical interaction that is linearly proportional to the mechanical position. The resulting square-displacement measurement strength is compared to that attainable in the dispersive case that has a direct interaction with the mechanical-displacement squared. An experimental protocol and parameter set are discussed for the generation and observation of non-Gaussian states of motion of the mechanical element.
\end{abstract}

DOI: 10.1103/PhysRevX.1.021011

\section{INTRODUCTION}

Currently the main approaches to cavity optomechanics [1] can be divided into two categories-reflective and dispersive. In each approach the mechanical and optical degrees of freedom are coupled via radiation pressure and the dependence of the cavity resonance frequency on the mechanical position. The first approach is depicted in Fig. 1(a), where the optical field is reflected from a mechanical element and the change in cavity frequency and hence interaction Hamiltonian are linearly proportional to the mechanical position. Optomechanical realizations of this approach include deformable Fabry-Perot cavities and optical whispering-gallery-mode resonators, which are discussed in Ref. [1]. The second approach is depicted in Fig. 1(b), where a mechanical element is positioned within an optical field and partial reflection from both sides gives rise to a dispersive interaction. In this arrangement, the cavity frequency varies periodically with mechanical displacement. This can be used to give a linear or quadratic position-dependent change in the cavity frequency if the mechanical element is positioned at an antinode or node of the field, respectively. The flexibility to select between linear or quadratic displacement coupling provides considerable versatility and thus dispersive optomechanics is an exciting candidate to observe and explore quantummechanical phenomena of macroscopic resonators. Optomechanical realizations of this approach utilize a dielectric membrane [2] or trapped cold atoms [3], positioned within an optical cavity, and experimental work is underway to realize this with an optically trapped microsphere [4]. The quadratic mechanical-position coupling offered by dispersive optomechanics provides a route to

Published by the American Physical Society under the terms of the Creative Commons Attribution 3.0 License. Further distribution of this work must maintain attribution to the author(s) and the published article's title, journal citation, and DOI. observe quantization in mechanical energy [2]. Moreover, such quadratic coupling can also be used for cooling and squeezing of the mechanical element [5] and it can be enhanced by using additional optical spatial modes, which even allows for quartic interaction [6].

In this paper, a scheme is presented that allows measurement of the mechanical displacement squared using an optomechanical interaction that is linear with the mechanical position. Here, optical pulses that are short compared to a mechanical period are used and the square-displacement coupling is obtained by exploiting the nonlinear optical dependence of the interaction. This interaction has been linearized in much of the present literature, but continuous nonlinear optomechanical interaction has recently been studied resulting in nonclassical states of light [7] and of the mechanical oscillator [8]. Also, working beyond the linear regime has been proposed for non-Gaussian quantum-state preparation of a collective spin variable [9]. The optomechanical setup considered here is shown in Fig. 1(c), where an optical pulse in a coherent state interacts with an optomechanical system and is then measured via homodyne detection. Following the interaction, Wigner reconstruction of the optical subsystem of the optomechanical entangled state, would yield a "scimitar state" shown in Fig. 1(d). The form of this optical state can be understood as the mechanical position fluctuations (including quantum fluctuations) rotate the optical field. For small rotations, one sees from Fig. 1(d) that measurement of the optical phase quadrature allows for a measurement of the mechanical position. However, of particular interest here, measurement of the amplitude quadrature may give outcomes that could have resulted from two distinct mechanical positions. This is due to an effective displacement-squared coupling, which can be used for non-Gaussian state preparation. In Ref. [10] it was discussed how measurement of the optical phase quadrature can be used to perform quantum-state tomography of the motional state of the mechanical resonator and generate conditional squeezed mechanical states. 
(a)

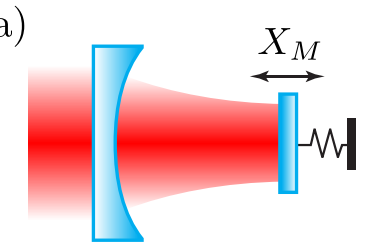

$H_{\text {int }} \propto a^{\dagger} a X_{M}$

(c)

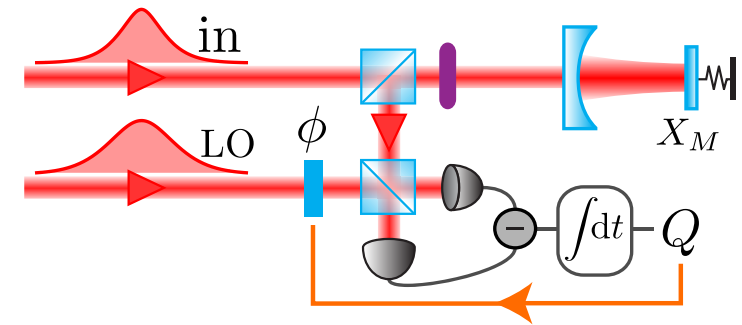

(d)

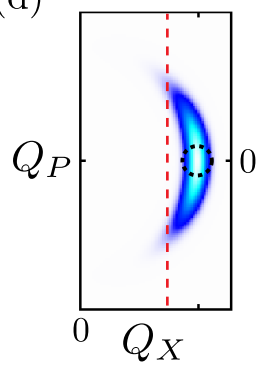

(e)

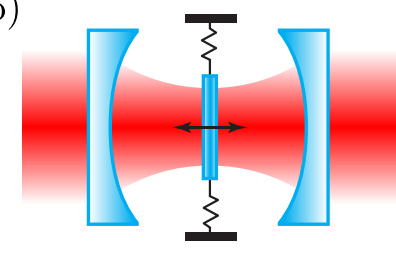

$H_{\text {int }} \propto a^{\dagger} a X_{M}^{2}$

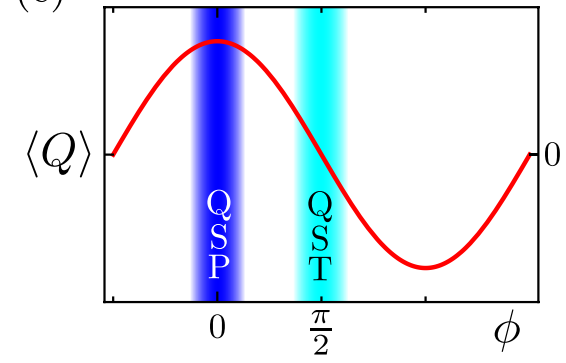

FIG. 1. Cavity optomechanics is currently realized using (a) reflective and (b) dispersive approaches. The interaction in the former is proportional to mechanical displacement $X_{M}$, however, in the latter the interaction can be tuned to being proportional to $X_{M}$ or $X_{M}^{2}$. Pulses of light may be used to probe and manipulate the motional state of the mechanical resonator. The optical setup considered here (c) is a pulse incident upon an optomechanical system with an interaction proportional to $X_{M}$ and then an optical quadrature measurement performed via homodyne detection. Following the interaction, the Wigner function of the optical field (d) is scimitar shaped due to mechanical-position-induced optical rotations. The distribution of the initial coherent state is indicated by the dashed circle. (The parameters for this plot were chosen to exaggerate the curvature.) For small rotations, the phase quadrature $Q_{P}$ is proportional to $X_{M}$ and the amplitude quadrature carries $X_{M}^{2}$ information. The amplitude-quadrature measurement outcome indicated by the red dashed line may have originated from two distinct mechanical positions, which provides a means for superposition preparation. By choosing the phase in (e) homodyne detection one can use the amplitude quadrature for $X_{M}^{2}$ measurements and quantum-state preparation or use the phase quadrature for $X_{M}$ measurements to perform quantum-state tomography.

Thus, the possibility to select between displacement and displacement-squared measurements provides the tools to generate non-Gaussian quantum states of the mechanical resonator and perform state reconstruction simply by choosing the phase in the homodyne interferometer as is shown in Fig. 1(e).

\section{MODEL}

The optomechanical Hamiltonian with linear mechanical position coupling in the optical rotating frame at the cavity frequency including a coherent resonant drive is

$$
\frac{H_{\text {lin }}}{\hbar}=\omega_{M} b^{\dagger} b-g_{\operatorname{lin}} \sqrt{2} a^{\dagger} a X_{M}-i \sqrt{2 \kappa N_{p}} \alpha_{\text {in }}\left(a-a^{\dagger}\right),
$$

where the optomechanical-coupling rate, which is realization dependent, is of the form $g_{\text {lin }}=\omega_{L} x_{0} / L$. The cavity field's resonance frequency, annihilation operator, and amplitude decay rate are $\omega_{L}, a$, and $\kappa$, respectively, and $L$ is the cavity length. The mechanical zero-point extension is $x_{0}=\sqrt{\hbar / 2 m \omega_{M}}$, where $\omega_{M}, b, m$, and $X_{M}\left(P_{M}\right)$ are the mechanical element's eigenfrequency, annihilation operator, effective mass, and position (momentum) quadrature (operator), respectively, where a single mechanical mode is considered. The input pulse has mean photon number $N_{p}$ and is described by $\alpha_{\text {in }}$, the normalized envelope, i.e., $\int d t \alpha_{\mathrm{in}}^{2}(t)=1$, which is assumed real.

During the interaction, which is short with respect to a mechanical period, thus requiring $\kappa \gg \omega_{M}$, the mechanical position is considered constant and the optical and mechanical equations of motion can be solved independently of one another. Immediately after the pulse interaction, the mechanical position is unchanged, i.e., $X_{M}^{\text {out }}=X_{M}^{\text {in }}$; however, optomechanical entanglement is generated and correlations are established between the mechanical momentum and the optical intensity, $P_{M}^{\text {out }}=$ $P_{M}^{\text {in }}+\sqrt{2} g_{\text {lin }} \int d t a^{\dagger} a$.

The intracavity field evolves during the nonlinear optomechanical interaction according to

$$
\frac{d a}{d t}=\left(i g_{\operatorname{lin}} \sqrt{2} X_{M}-\kappa\right) a+\sqrt{2 \kappa}\left(\sqrt{N_{p}} \alpha_{\mathrm{in}}+a_{\mathrm{in}}\right),
$$

where the field is rotated in proportion to the mechanical position and $a_{\text {in }}$ is the optical input noise. This can be immediately solved exactly [11], however, in this work the solution is approximated as the rotation is assumed small and the mean of the field is

$$
\frac{\langle a(t)\rangle}{\sqrt{N_{p}}} \simeq \alpha_{0}(t)+i \frac{g_{\text {lin }}}{\kappa} \alpha_{1}(t)\left\langle X_{M}\right\rangle-\frac{g_{\text {lin }}^{2}}{\kappa^{2}} \alpha_{2}(t)\left\langle X_{M}^{2}\right\rangle,
$$

where the dimensionless temporal mode functions $\alpha_{0,1,2}$ are introduced [12]. The phase quadrature of the intracavity field contains information on the mechanical displacement, and the amplitude quadrature carries information of the mechanical-displacement squared [Fig. 1(e)]. Measurements of these quadratures can be performed by time-domain homodyne detection of the output field $a_{\text {out }}=$ $\sqrt{2 \kappa} a-a_{\text {in }}$. Homodyning the amplitude quadrature is described by $Q_{X}=\sqrt{2} \int d t \alpha_{\mathrm{LO}}(t) X_{L}^{\text {out }}(t)$, where $X_{L}^{\text {out }}=$ $2^{-1 / 2}\left(a_{\text {out }}+a_{\text {out }}^{\dagger}\right)$ (similarly $Q_{P}$ describes phase-quadrature detection). For an optimal measurement of $X_{M}^{2},\left(X_{M}\right)$ 
one chooses the local oscillator pulse $\alpha_{\mathrm{LO}}$ to have an amplitude directly proportional to $\alpha_{2},\left(\alpha_{1}\right)$. The mean of the amplitude-quadrature measurement is $\left\langle Q_{X}\right\rangle=Q_{X}^{(0)}$ $\chi_{X}\left\langle X_{M}^{2}\right\rangle$, where the first term is the contribution from $\alpha_{0}$ and $\chi_{X}$ is the square-displacement measurement strength. For convenience, the outcome of the homodyne measurement is rewritten as $\Delta Q_{X}=Q_{X}^{(0)}-Q_{X}$. The optimal single-pulsed measurement of $X_{M}$ is achieved with an input drive with a Lorentzian spectrum, which matches the natural decay of the cavity [10]. The square-displacement measurement strength is optimal when $\alpha_{\text {in }}^{2}(\omega)=(3 \pi)^{-1} 8 \kappa^{5} /\left(\kappa^{2}+\omega^{2}\right)^{3}$, which is not Lorentzian due to the higher-order nature of the interaction considered here. This gives $\chi_{X}=$ $\sqrt{42 N_{p}} g_{\text {lin }}^{2} / \kappa^{2}$.

This kind of pulsed interaction and measurement is well suited to being described with the use of measurement operators as outcome probabilities, and conditional mechanical states can be readily determined [13]. Homodyne detection of the amplitude quadrature has the outcome probability density $\operatorname{Pr}\left(\Delta Q_{X}\right)=\operatorname{Tr}_{M}\left(\Upsilon_{X}^{\dagger} \Upsilon_{X} \rho_{M}^{\text {in }}\right)$, where $Y_{X}$ is the corresponding measurement operator. In this pulsed regime $\Delta Q_{X}$ has mechanical dependence only on $X_{M}$, which allows $Y_{X}^{\dagger} Y_{X}$ to be interpreted as an outcome probability density conditioned on a mechanical position. For the coherent optical drive considered here one obtains

$$
\Upsilon_{X}\left(X_{M}, \Delta Q_{X}\right)=\pi^{-1 / 4} e^{i \Omega_{\operatorname{lin}} X_{M}} \exp \left[-\frac{1}{2}\left(\Delta Q_{X}-\chi_{X} X_{M}^{2}\right)^{2}\right],
$$

where the mean momentum transfer is $\Omega_{\text {lin }}=$ $(5 \sqrt{2} / 3) N_{p} g_{\text {lin }} / \kappa$.

\section{COMPARISON TO THE DISPERSIVE QUADRATIC INTERACTION}

Before proceeding to a discussion of the mechanical states of motion that can be prepared with $Y_{X}$, the square-displacement measurement scheme introduced above is compared with the dispersive case. The Hamiltonian from Ref. [2] for optomechanical systems with a dispersive element positioned so that the cavity frequency varies quadratically with the position of the element, in the optical rotating frame at resonance, including the drive is

$\frac{H_{\mathrm{sq}}}{\hbar}=\omega_{M} b^{\dagger} b+g_{\mathrm{sq}} a^{\dagger} a X_{M}^{2}-i \sqrt{2 \kappa N_{p}} \alpha_{\mathrm{in}}\left(a-a^{\dagger}\right)$,

where the quadratic-optomechanical coupling rate is $g_{\text {sq }}=\left(16 \pi^{2} c x_{0}^{2} / L \lambda^{2}\right) \sqrt{2(1-r)}, r$ is the (field) reflectivity of the dispersive element, and $\lambda$ is the optical wavelength. The phase quadrature of an optical pulse incident upon such an optomechanical system will be displaced in proportion to $X_{M}^{2}$, and it is readily shown that for a homodyne measurement of the phase quadrature with outcome
$Q_{P}$ the measurement operator is $Y_{\mathrm{sq}}=\pi^{-1 / 4} e^{-i \Omega_{\mathrm{sq}} X_{M}^{\text {in }} X_{M}} \times$ $\exp \left[-\frac{1}{2}\left(Q_{P}+\chi_{\mathrm{sq}} X_{M}^{2}\right)^{2}\right]$, which has recently been used in Ref. [14]. After pulse-shape optimization, $\Omega_{\mathrm{sq}}=$ $3 N_{p} g_{\mathrm{sq}} / \kappa$ and $\chi_{\mathrm{sq}}=\sqrt{10 N_{p}} g_{\mathrm{sq}} / \kappa$. Comparing the measurement strengths for the dispersive direct $X_{M}^{2}$ interaction and the effective $X_{M}^{2}$ coupling from the linear interaction for identical $N_{p}$ and $\lambda$ gives

$$
\frac{\chi_{X}}{\chi_{\mathrm{sq}}} \simeq \frac{1}{\pi} \frac{\mathcal{F}_{\mathrm{lin}}^{2}}{\mathcal{F}_{\mathrm{sq}}} \frac{x_{\mathrm{lin}}^{2}}{x_{\mathrm{sq}}^{2}} \frac{1}{\sqrt{2(1-r)}},
$$

where the cavity finesses and mechanical zero-point extensions are distinguished by subscripts for the two optomechanical cases. Remarkably, using the optomechanical interaction that is linearly proportional to $X_{M}$ and optical amplitude-quadrature measurements allows for $X_{M}^{2}$ measurements that are stronger than that available with the direct $X_{M}^{2}$ interaction in dispersive optomechanics by approximately the cavity finesse. This, in combination with the measurement-based selectability between linear or quadratic couplings offered here, is the main result of this work.

\section{EXPERIMENTAL PROTOCOL AND DISCUSSION}

Jacobs and colleagues discussed the preparation of superposition of the position of a mechanical resonator via $X_{M}^{2}$ measurements [15]. This work has recently been extended to include feedback control of the superposition separation [16]. Such benchmark quantum states show striking differences between classical and quantum behavior and are thus highly sought experimentally to study the quantum-mechanical properties of macroscopic objects [17-19]. In the following, an experimental protocol and a parameter set are discussed to prepare and observe the spatial superposition of a massive mechanical resonator using the nonlinear interaction and measurement $Y_{X}$. A measurement on a variety of experimentally accessible initial states is considered, and the resulting conditional and unconditional mechanical states of motion are determined.

As the spectrum of measurement outcomes is continuous, it is not experimentally possible to postselect from many experimental runs on a single measurement outcome. Instead, a window must be used. The mechanical state conditioned on outcomes occurring in the window $\Delta Q_{X} \pm$ $w / 2$ (labeled by $w$ ) is

$$
\rho_{M}^{(w)}=\frac{1}{\operatorname{Pr}(w)} \int_{w} d \Delta Q_{X}^{\prime} \Upsilon_{X}\left(\Delta Q_{X}^{\prime}\right) \rho_{M}^{\mathrm{in}} \Upsilon_{X}^{\dagger}\left(\Delta Q_{X}^{\prime}\right),
$$

where $\operatorname{Pr}(w)=\int_{w} d \Delta Q_{X} \operatorname{Pr}\left(\Delta Q_{X}\right)$ is the probability of obtaining an outcome in the window. The mean measurement outcome for a mechanical thermal state with thermal occupation $\bar{n}$ is $\left\langle\Delta Q_{X}\right\rangle=\chi_{X}(1 / 2+\bar{n})$. As the mean is greater than zero, some insight is gained into the form of 
$\operatorname{Pr}\left(\Delta Q_{X}\right)$, which is a non-Gaussian function with a large wing for positive outcomes that increases for a larger mechanical position variance.

In Fig. 2 the action of $Y_{X}$ is considered on three mechanical Gaussian states: the ground state, a thermal state, and a momentum-squeezed state. One may suspect that quite a narrow window for conditioning must be used in order to achieve significant coherence between the superposition components, however, conditional mechanical states, prepared from high-purity initial states, show strong quantum coherence even for relatively large conditioning windows. For example, the conditional mechanical state shown in Fig. 2(b) exhibits strong Wigner negativity even for $w=0.8$, which allows the use of $15 \%$ of the measurement outcomes. This plot also reveals the interesting feature that the negative regions are "curled around" positive regions, a feature which is not seen in the more commonly studied superposition of coherent states. This arises due to the population components having an asymmetric distribution about their peaks, specifically, there is a broader
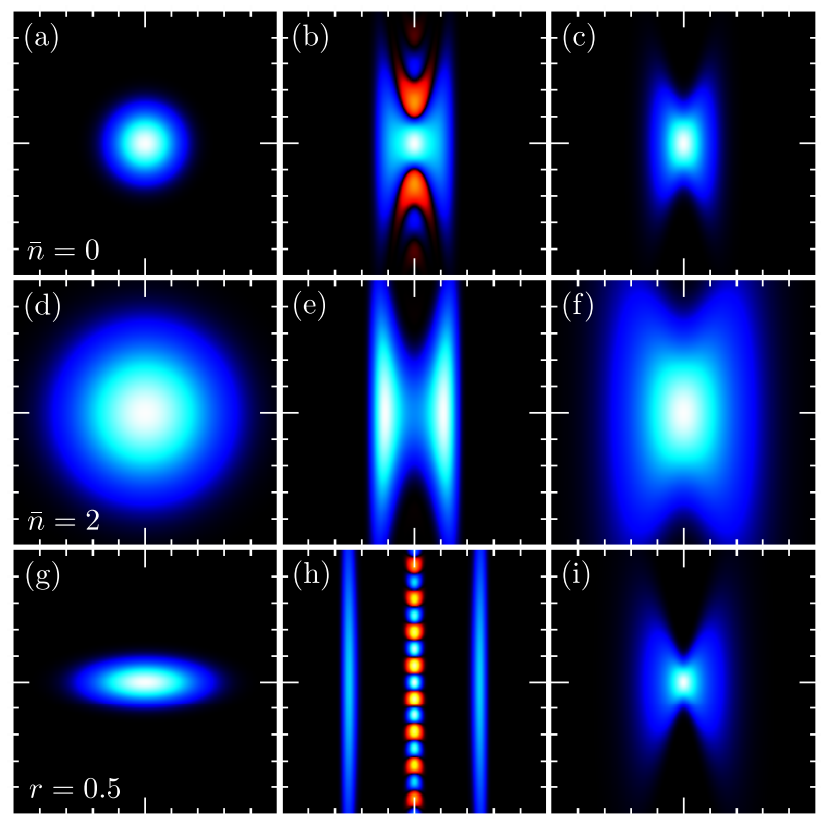

FIG. 2. Mechanical Wigner functions of initial states (left), conditional states (center), and unconditional states (right). ( $X_{M}$ is the horizontal axis, $P_{M}$ is the vertical axis. The plot range is \pm 5 for all axes. Color scale: black is for zero magnitude, blue for positive values, and red for negative values.) The initial states are the ground state, $\bar{n}=0$ (a), a thermal state with $\bar{n}=2$ (d), and a momentum-squeezed vacuum state with squeezing parameter $r=0.5(\mathrm{~g})$. Conditional states prepared with $\Upsilon_{X}$ acting on the corresponding initial states with $\chi_{X}=1, \Delta Q_{X}=$ $1.5, w=0.8$ are shown in (b) and (e) and $\Delta Q_{X}=6.4$ has been used in (h). The probabilities of obtaining an outcome in the windows used above are: (b) $14.9 \%$, (e) $14.5 \%$, and (h) $1.1 \%$. Note the disappearance of negativity - a quantum-to-classical transition-for initial thermal occupation (e) and if the measurement outcomes are ignored (c), (f), (h). wing nearer $X_{M}=0$ and a sharper edge on the other side. This form of the population components is more clearly seen in Fig. 2(e), which is the conditional mechanical state starting from a low-occupation thermal state. When the population components have a more symmetric $X_{M}$ distribution about their peak, the interferences no longer curl as strongly, as is seen in Fig. 2(h), the conditional state starting from a squeezed state.

Since a measurement of the optical amplitude quadrature erases all the linear-displacement information gained during the interaction and $\left\langle\Delta Q_{X}\right\rangle>0$, the unconditional (i.e., all measurement outcomes are ignored) mechanical state, $\rho_{M}^{\text {out }}=\int_{-\infty}^{\infty} d \Delta Q_{X} \Upsilon_{X} \rho_{M}^{\text {in }} \Upsilon_{X}^{\dagger}$, is also non-Gaussian, however, mixed.

The superposition separation $\delta$ is defined as the distance between the maxima of the two population components. This depends on the initial mechanical distribution, the measurement outcome, and the square-displacement measurement strength. For Gaussian initial mechanical states with a standard deviation $\sigma$ in their position spread, the superposition separation is

$$
\delta=\frac{\sqrt{4 \Delta Q_{X} \chi_{X}-\sigma^{-2}}}{\chi_{X}} .
$$

Experimental progress in optomechanics is steadily approaching the regime where the important parameter $g_{\text {lin }} / \kappa$, which quantifies the mechanical momentum displacement by a single photon (for $\kappa \gg \omega_{M}$ ), approaches unity. In this work $\chi_{X}$ scales with the square of this parameter and the pulsed position-measurement strength for mechanical quantum-state tomography [10] scales linearly with this parameter. In present-day experiments [20], $g_{\operatorname{lin}} / \kappa \ll 1$, which this work overcomes by utilizing large coherent amplitudes in order to achieve sufficient coupling to prepare and observe non-Gaussian mechanical states of motion. To ensure a short interaction, the cavity decay rate is chosen as $\kappa=10^{3} \omega_{M}$, which for a desired finesse sets the cavity length required. In Table I a list of parameters is provided for a deformable Fabry-Perot optomechanical system with a kHz-scale mechanical resonator.

The protocol for quantum-state preparation and quantum-state tomography comprises three steps: (i) an initialization stage of mechanical precooling and/or squeezing. Since $\kappa \gg \omega_{M}$ is required here and lowfrequency mechanical resonators are considered, activefeedback cooling is most suitable [21,22]. Alternatively, in this regime, squeezing and purification can be achieved with the use of conditional measurements [10]. Additionally, squeezing can be achieved by applying a parametric modulation to the mechanical device [23]. (ii) Following this, an optical pulse is injected into the optomechanical cavity to realize $Y_{X}$ and the measurement outcome is recorded. At this point, the mechanical oscillator has gained the momentum $\Omega_{\text {lin }}$, which after one quarter of a period of free evolution shifts the cavity 
TABLE I. An experimentally accessible set of parameters to achieve unity square-displacement measurement strength.

\begin{tabular}{lcc}
\hline \hline Optical wavelength: & $\lambda$ & $1064 \mathrm{~nm}$ \\
Mechanical effective mass: & $m$ & $40 \mathrm{ng}$ \\
Mechanical eigenfrequency: & $\omega_{M} / 2 \pi$ & $2 \mathrm{kHz}$ \\
Cavity finesse: & $\mathcal{F}$ & $5 \times 10^{4}$ \\
Photon number per pulse: & $N_{p}$ & $1.7 \times 10^{9}$ \\
Cavity length: & $L$ & $750 \mu \mathrm{m}$ \\
Mechanical ground-state size: & $x_{0}$ & $10 \mathrm{fm}$ \\
Optomechanical coupling: & $g_{\operatorname{lin}} / 2 \pi$ & $3.8 \mathrm{kHz}$ \\
Single-photon strength: & $g_{\operatorname{lin}} / \kappa$ & $1.9 \times 10^{-3}$ \\
Separation $\left(\bar{n}=0, \Delta Q_{X}=1.5\right):$ & $\delta$ & 2.0 \\
\hline \hline
\end{tabular}

resonance frequency by $\Delta \omega_{\Omega}=g_{\text {lin }} \sqrt{2} \Omega_{\text {lin }}$. As this can be much larger than $\kappa$ any subsequent pulse will not resonantly drive the cavity. In order to overcome this, a twopulse preparation sequence can be used where a second pulse follows after half a mechanical period of free evolution to cancel the mean momentum gained by the resonator. In this case, one applies $Y_{X}$ twice where both outcomes are recorded, thus strengthening the measurement of $X_{M}^{2}$. This procedure requires a good degree of optical amplitude stability, which is necessary for $Y_{X}$ measurements anyway. During the free evolution, the appropriate master equation is solved to determine the mechanical state immediately prior to the second measurement. However, as discussed below, given the parameters considered here, the coupling to the mechanical bath is not expected to play a strong role during this time scale. (iii) With the resonator state near the origin of phase space, quantum-state tomography, as discussed in Ref. [10], is now performed. This is achieved by later injecting a subsequent pulse with the local oscillator phase switched to measure the optical phase quadrature as in Fig. 1(e). Repeating this protocol many times and postselecting the measurement outcomes $\Delta Q_{X}$ within the desired window provides a powerful experimental platform to generate and fully reconstruct a non-Gaussian state of motion of a mechanical resonator.

In order to prepare mechanical superposition states with $\Upsilon_{X}$ there needs to be a sufficient mechanical-displacementinduced optical rotation such that two distinct positions give the same amplitude-quadrature outcome. This is best achieved if the mechanical mean position gives zero rotation. For mechanical states that have a nonzero mean, which could have been conditionally prepared with a prior pulse [10], non-Gaussian state preparation and tomography can be performed by providing a feedback phase shift [indicated by the arrow in Fig. 1(c)] to rotate the optical scimitar to be centered about the $Q_{X}$ axis, as in Fig. 1(d). Additionally, it is noted that for optical rotation beyond that considered in (3), existing experimental calibration procedures and the interpretation of optical phase measurements will require modification to take the optomechanical nonlinearity into account.
Studying the decoherence of quantum superposition in a mechanical resonator is important to determine the feasibility of optomechanical systems as components for quantum-information applications. Proposals for such applications are numerous and include quantum memory [24], optomechanically mediated qubit-light transduction [25], and coherent optical wavelength conversion [26], to name a few. There is much literature on the topic of environmental coupling and decoherence [27], so no detailed discussion will be provided here. However, in the context of this proposal, what is important is the parameter $\bar{n} / Q$, where $Q$ is the mechanical quality factor. This parameter quantifies the rate of rethermalization normalized to the mechanical frequency and must be much less than unity for studying the evolution of quantum-mechanical phenomena over the time scale $\omega_{M}^{-1}$. A temperature of $25 \mathrm{mK}$ accessible with dilution refrigeration and a $Q=$ $5 \times 10^{6}$ give $\bar{n} / Q=0.05$ using the mechanical frequency above. With the full quantum-state tomography available here, this scheme allows the dynamics of mechanical superposition states to be measured, which may be used to characterize the couplings responsible for decoherence, thus allowing for improved mechanical device engineering.

Furthermore, the significant mass involved in the spatial superposition offers a parameter regime that allows for an experimental test of collapse models. Very recent proposals in matter-wave interferometry [14,28], which also consider the use of filtering-type operations to generate superposition, may provide the ability to test continuous spontaneous localization [29]. The mechanical resonator parameters considered here are not suitable for testing continuous spontaneous localization predominantly because the superposition separation is small [30]. However, the separation can be larger than the distribution of the mass contained within the nucleus and so this can be used to test gravitational collapse [31]. For example, using the parameters above $\left(\delta=2.0, x_{0}=10 \mathrm{fm}\right)$ the separation is approximately $28 \mathrm{fm}$ and the diameter of a ${ }^{28} \mathrm{Si}$ nucleus is approximately $8 \mathrm{fm}$. It may be useful in such an investigation to start with an initial squeezed mechanical state, as is considered in Figs. 2(g)-2(i), as one can study a larger range of superposition separations as the probability density of measurement outcomes is broader.

\section{CONCLUSION}

This work has provided a means to measure the displacement or displacement squared of a mechanical resonator using the optomechanical interaction linearly proportional to the mechanical displacement by simply changing the phase in optical homodyne measurement. Displacement-squared measurements have so far been predominantly considered in dispersive optomechanics; however, the optimal square-displacement measurement strength obtained in the scheme introduced here can be 
significantly stronger than that available in dispersive optomechanics as it scales more favorably with the cavity finesse. This opens the possibility that optomechanics with an interaction Hamiltonian that is linear with the mechanical position may also provide a route to observe mechanical-energy quantization, as was considered in [2]. Moreover, as was proposed in [15], with an $X_{M}^{2}$ coupling to a mechanical resonator one can prepare a superposition of positions via measurement. This, applied to the $X_{M}^{2}$ coupling achieved here and combined with the ability to perform mechanical state tomography with time [10], provides an alternative to Refs. [17,19] to generate the superposition of a mechanical resonator without the need for large single-photon mechanical displacement $g_{\text {lin }} / \kappa$. Such mechanical superposition states are important to investigate experimentally in order to determine the feasibility of mechanical resonators as elements in quantuminformation applications and to explore decoherence mechanisms arising from environment interaction or, for example, gravitationally induced collapse.

\section{ACKNOWLEDGMENTS}

M. R. V. acknowledges support from the FWF Doctoral Programme CoQuS (W 1210), the Austrian Academy of Sciences, and gratefully acknowledges discussion with Markus Aspelmeyer, Gerard J. Milburn, and Igor Pikovski.

[1] T. J. Kippenberg and K. J. Vahala, Cavity Optomechanics: Back-Action at the Mesoscale, Science 321, 1172 (2008); I. Favero and K. Karrai, Optomechanics of Deformable Optical Cavities, Nat. Photon. 3, 201 (2009); F. Marquardt and S. T. Girvin, Optomechanics, Physics 2, 40 (2009); M. Aspelmeyer, S. Groeblacher, K. Hammerer, and N. Kiesel, Quantum Optomechanics-Throwing a Glance, JOSA B 27, A189 (2010).

[2] J. D. Thompson, B. M. Zwickl, A. M. Jayich, F. Marquardt, S.M. Girvin, and J.G.E. Harris, Strong Dispersive Coupling of a High-Finesse Cavity to a Micromechanical Membrane, Nature (London) 452, 72 (2008).

[3] T. P. Purdy, D. W. C. Brooks, T. Botter, N. Brahms, Z.-Y. Ma, and D.M. Stamper-Kurn, Tunable Cavity Optomechanics with Ultracold Atoms, Phys. Rev. Lett. 105, 133602 (2010).

[4] See, for example, T. Li, S. Kheifets, and Mark G. Raizen, Millikelvin Cooling of an Optically Trapped Microsphere in Vacuum, Nature Phys. 7, 527 (2011).

[5] A. Nunnenkamp, K. Borkje, J. G. E. Harris, and S. M. Girvin, Cooling and Squeezing via Quadratic Optomechanical Coupling, Phys. Rev. A 82, 021806(R) (2010).

[6] J. C. Sankey, C. Yang, B. M. Zwickl, A. M. Jayich, and J.G.E. Harris, Strong and Tunable Nonlinear Optomechanical Coupling in a Low-Loss System, Nature Phys. 6, 707 (2010).

[7] P. Rabl, Photon Blockade Effect in Optomechanical Systems, Phys. Rev. Lett. 107, 063601 (2011).
[8] A. Nunnenkamp, K. Borkje, and S. M. Girvin, SinglePhoton Optomechanics, Phys. Rev. Lett. 107, 063602 (2011).

[9] S. Massar and E. S. Polzik, Generating a Superposition of Spin States in an Atomic Ensemble, Phys. Rev. Lett. 91, 060401 (2003).

[10] M. R. Vanner, I. Pikovski, G. D. Cole, M. S. Kim, C. Brukner, K. Hammerer, G. J. Milburn, and M. Aspelmeyer, Pulsed Quantum Optomechanics, Proc. Natl. Acad. Sci. U.S.A. 108, 16182 (2011).

[11] This Hamiltonian has been previously used to model wavemixing processes, which allow for the quantum nondemolition measurement of the number squared, see G. J. Milburn and D. F. Walls, Quantum Nondemolition Measurements via Quantum Counting, Phys. Rev. A 28, 2646 (1983).

[12] The cavity integrates the input drive and phase accumulates proportional to $X_{M}$ and $X_{M}^{2}$ with the dimensionless envelopes: $\alpha_{0}(t)=\sqrt{2 \kappa} \int_{-\infty}^{t} d t^{\prime} e^{-\kappa\left(t-t^{\prime}\right)} \alpha_{\text {in }}\left(t^{\prime}\right)$, $\alpha_{1}(t)=\sqrt{2 \kappa} \int_{-\infty}^{t} d t^{\prime} e^{-\kappa\left(t-t^{\prime}\right)} \kappa \sqrt{2}\left(t-t^{\prime}\right) \alpha_{\text {in }}\left(t^{\prime}\right), \quad \alpha_{2}(t)=$ $\sqrt{2 \kappa} \int_{-\infty}^{t} d t^{\prime} e^{-\kappa\left(t-t^{\prime}\right)} \kappa^{2}\left(t-t^{\prime}\right)^{2} \alpha_{\text {in }}\left(t^{\prime}\right)$, respectively.

[13] For a recent discussion on generalized quantum measurement see, for example, H. M. Wiseman and G. J. Milburn, Quantum Measurement and Control (Cambridge University Press, Cambridge, 2010).

[14] O. Romero-Isart, A. C. Pflanzer, F. Blaser, R. Kaltenbaek, N. Kiesel, M. Aspelmeyer, and J. I. Cirac, Large Quantum Superpositions and Interference of Massive NanometerSized Objects, Phys. Rev. Lett. 107, 020405 (2011).

[15] K. Jacobs, L. Tian, and J. Finn, Engineering Superposition States and Tailored Probes for Nanoresonators via Open-Loop Control, Phys. Rev. Lett. 102, 057208 (2009).

[16] K. Jacobs, J. Finn, and S. Vinjanampathy, Real-Time Feedback Control of a Mesoscopic Superposition, Phys. Rev. A 83, 041801(R) (2011).

[17] S. Bose, K. Jacobs, and P. Knight, Scheme to Probe the Decoherence of a Macroscopic Object, Phys. Rev. A 59, 3204 (1999).

[18] A. D. Armour, M. P. Blencowe, and K. C. Schwab, Entanglement and Decoherence of a Micromechanical Resonator via Coupling to a Cooper-Pair Box, Phys. Rev. Lett. 88, 148301 (2002).

[19] W. Marshall, C. Simon, R. Penrose, and D. Bouwmeester, Towards Quantum Superpositions of a Mirror, Phys. Rev. Lett. 91, 130401 (2003).

[20] Examples of experiments with comparatively large $g_{\text {lin }} / \kappa$ can be found in the following: A. H. Safavi-Naeini, T. P. Mayer Alegre, M. Winger, and O. Painter, Optomechanics in an Ultrahigh-Q Two-Dimensional Photonic Crystal Cavity, Appl. Phys. Lett. 97, 181106 (2010); J. D. Teufel, Dale Li, M.S. Allman, K. Cicak, A.J. Sirois, J.D. Whittaker, and R.W. Simmonds, Circuit Cavity Electromechanics in the Strong-Coupling Regime, Nature (London) 471, 204 (2011).

[21] C. Genes, D. Vitali, P. Tombesi, S. Gigan, and M. Aspelmeyer, Ground-State Cooling of a Micromechanical Oscillator: Comparing Cold Damping and Cavity-Assisted Cooling Schemes, Phys. Rev. A 77, 033804 (2008).

[22] J. Zhang, Y.-x. Liu, and F. Nori, Cooling and Squeezing the Fluctuations of a Nanomechanical Beam by Indirect Quantum Feedback Control, Phys. Rev. A 79, 052102 (2009). 
[23] See, for example, A. Mari and J. Eisert, Gently Modulating Optomechanical Systems, Phys. Rev. Lett. 103, 213603 (2009).

[24] J. Zhang, K. Peng, and S. L. Braunstein, Quantum-State Transfer from Light to Macroscopic Oscillators, Phys. Rev. A 68, 013808 (2003).

[25] K. Stannigel, P. Rabl, A. S. Sorensen, P. Zoller, and M. D. Lukin, Optomechanical Transducers for Long-Distance Quantum Communication, Phys. Rev. Lett. 105, 220501 (2010).

[26] L. Tian and H. Wang, Optical Wavelength Conversion of Quantum States with Optomechanics, Phys. Rev. A 82, 053806 (2010).

[27] See M. Schlosshauer, Decoherence and the Quantum-toClassical Transition (Springer, New York, 2007) for a recent discussion.
[28] S. Nimmrichter, P. Haslinger, K. Hornberger, and M. Arndt, Concept of an Ionizing Time-Domain Matter-Wave Interferometer, New J. Phys. 13, 075002 (2011); S. Nimmrichter, K. Hornberger, P. Haslinger, and M. Arndt, Testing Spontaneous Localization Theories with MatterWave Interferometry, Phys. Rev. A 83, 043621 (2011).

[29] See, for example, S. L. Adler and A. Bassi, Is Quantum Theory Exact?, Science 325, 275 (2009) and references therein.

[30] A. Bassi, E. Ippoliti, and S. L. Adler, Towards Quantum Superpositions of a Mirror: An Exact Open Systems Analysis, Phys. Rev. Lett. 94, 030401 (2005).

[31] L. Diósi, Models for Universal Reduction of Macroscopic Quantum Fluctuations, Phys. Rev. A 40, 1165 (1989); R. Penrose, On Gravity's Role in Quantum State Reduction, Gen. Relativ. Gravit. 28, 581 (1996). 